\title{
Teoría de Resolución de Conflictos de Johan Galtung para la Implementación de la Cátedra de la Paz
}

\author{
Johan Galtung's Conflict Resolution Theory for the Implementation of the Chair of \\ Peace
}

Dayanna Zuley Cely-Fuentes ${ }^{1}$

Recibido: 25/junio/2021

Aceptado: $18 /$ septiembre/2021

Publicado: $24 /$ septiembre/2021

秋 País

${ }^{1}$ Colombia

\section{IIII. Institución}

${ }^{1}$ Universidad de Pamplona

\section{\Correo Eletrónico \\ 1DDayanacelis235@gmail.com}

\section{(D) ORCID}

${ }^{1}$ https://orcid.org/0000-0003-4701-8569

\section{Citar así: CFAPA / IEEE}

Cely-Fuentes, D. (2021). Teoría de Resolución de Conflictos de Johan Galtung para la Implementación de la Cátedra de la Paz. Revista Tecnológica-Educativa Docentes 2.0, 11(2), 48-56. https://doi.org/10.37843/rted.v11i2.251

D. Cely-Fuentes, "Teoría de Resolución de Conflictos de Johan Galtung para la Implementación de la Cátedra de la Paz ", RTED, vol. 11, n. ${ }^{\circ}$ 2, pp. 48-56, sep. 2021

\section{Resumen}

Propender por una cultura de paz en un país y en una región históricamente marcada por la violencia es un reto que debe iniciar desde las escuelas y frente a esta perspectiva el objetivo de esta investigación se centró en analizar la incidencia de la teoría de resolución de conflictos de Johan Galtung, la cual enuncia los conceptos de conflicto y paz, hace referencia a acciones concretas para disminuir o erradicar los conflictos para favorecer entornos de sana convivencia. La aplicación de esta teoría estuvo mediada por diversas estrategias pedagógicas y didácticas: página web Fanzineros por la paz, cartilla MI DIARIO, formularios y pruebas de diagnóstico y salida. La metodología utilizada fue de carácter cualitativo siendo la más precisa para investigar los fenómenos sociales en el que se persiguen determinados objetivos para así dar respuesta a algunos problemas concretos. La población con quienes se desarrolló la investigación se caracteriza por estar comprendida entre los 13 y 14 años de edad, es un grupo de 34 estudiantes. Como resultado se logró evidenciar una disminución en los índices de violencia escolar y por ende un mejoramiento de la convivencia escolar y de las relaciones humanas. La principal conclusión indica el restablecimiento de una cultura de paz, entendida como la apropiación de conocimientos y competencias ciudadanas para la construcción de una sana convivencia.

Palabras clave: Paz, educación para la paz, conflicto y violencia, resolución y transformación de conflictos.

\section{Abstract}

To strive for a culture of peace in a country and a region historically marked by violence is a challenge that must start from the schools. In light of this perspective, the objective of this research focused on analyzing the incidence of conflict resolution theory. Johan Galtung, which enunciates the concepts of conflict and peace, refers to concrete actions to reduce or eradicate conflicts to favor environments of healthy coexistence. Various pedagogical and didactic strategies mediated the application of this theory: Fanzineros for la Paz web page, MY DIARY booklet, diagnostic and exit forms, and tests. The methodology used was qualitative, being the most precise to investigate social phenomena in which specific objectives are pursued to respond to particular problems. The population with whom developed the research is between 13 and 14 years of age; it is a group of 34 students. As a result, it was possible to show a decrease in the rates of school violence and, therefore, an improvement in school coexistence and human relations. The main conclusion indicates the reestablishment of a culture of peace, understood as the appropriation of knowledge and citizen skills to construct a healthy coexistence.

Keywords: Conflict, transformation and conflict resolution, peace, education for peace. 


\section{Introducción}

Propender por una cultura de paz en un país y en una región históricamente marcada por la violencia es un reto que debe iniciar desde las escuelas, estas circunstancias plantean la necesidad de incluir en el currículo académico la cátedra de La Paz. Colombia, en aras de buscar la paz y el mejoramiento de la calidad de vida de todos los colombianos, de la mano del Ministerio de Educación Nacional, a través de la Ley 1732 de 2014 por la cual se establece la cátedra de la paz, obliga a todas las instituciones educativas del país a implementarla como un área del currículo, pero detrás de ello, no sólo está el ánimo de buscar paz, sino mejorar los procesos de convivencia escolar y disminuir o eliminar las cifras de intimidación, acoso, matoneo y violencia escolar.

Unido a la promoción de la Ley de convivencia escolar, la cátedra de la paz es la herramienta que el Estado colombiano crea como recurso para formar sujetos de paz y transformadores sociales que se articula en todos los niveles de educación, tocando diferentes temas que se ve implicados en las ciencias sociales y humanas, lo que permite una reflexión y contextualización de las problemáticas del conflicto armado colombiano, siendo estos de vital importancia para los nuevos retos que vienen para el país en términos de resocialización, resolución de conflictos y sana convivencia teniendo en cuenta que la transformación del país se da en medida que los colombianos y colombianas sean promotores de paz.

El autor elegido para fundamentar esta investigación, ha sido Johan Galtung (sociólogo, y matemático Noruego), y teniendo en cuenta su importancia en estudios de temas de paz, la teoría elegida para justificar esta investigación ha sido la teoría del conflicto, y de su mano, esta teoría se complementa con los postulados expuestos en las obras: paz por medios pacíficos, reconstrucción, reconciliación y reconciliación, y trascender y transformar, las cuales fueron tomadas como ejes importantes para abordar los problemas que dificultan los procesos de sana convivencia y fomentan el desarrollo de una cultura de paz.

Para Galtung (1984), la violencia es una manifestación de los conflictos no resueltos, es una privación de los Derechos Humanos hacia la vida, la felicidad y la prosperidad, y se representa como una disminución de los niveles de satisfacción de las necesidades, por tanto, los postulados de Chaux (2004), enuncian que las competencias ciudadanas son los conocimientos y las habilidades cognitivas, emocionales y comunicativas que hacen posible que las personas participen en la construcción de una sociedad democrática, pacífica e incluyente. Así mismo James (2018) propone que el compromiso de educar para la paz puede expresarse en trabajar por un proceso educativo que signifique contribuir a alejar el peligro de la guerra.

En la mayoría de las instituciones educativas a pesar del fomento de los valores y la promoción de la cultura de la paz, se presentan situaciones de violencia directa como agresiones físicas y verbales, uso de palabras soeces, discriminaciones $y$ situaciones de acoso. Partiendo de lo anterior, el objetivo de esta investigación se centró en desarrollar una cultura de paz desde la escuela, haciendo uso de la comunicación y el diálogo para resolver los conflictos, garantizando que estos no vuelvan a ocurrir, y proporcionando a los actores las herramientas necesarias para convertir los conflictos en oportunidades, para que quienes ayer y hoy se enfrentaban, sean mañana los mediadores y constructores de paz, y todo esto se logró a través de la comprensión de los postulados teóricos, los cuales permitieron el abordaje del proceso de enseñanza y aprendizaje para abrir las posibilidades a la comprensión y aplicación de esta investigación.

\section{Metodología}

Esta investigación se desarrolló con un enfoque cualitativo, y teniendo en cuenta que el estudio de la paz es un fenómeno social, este mismo permitió reconocer las clases de violencia y sus consecuencias para manifestar formas creativas de disminuirla y erradicarla. Por lo anterior, esta investigación permitió la 
comprensión del conjunto de cualidades que caracterizan al fenómeno de la paz y acercarse a la realidad social a partir de la utilización de datos no cuantitativos. Unido a ello, aplicar este enfoque permitió:

\section{Al investigador usar diversos} recursos para conocer las concepciones que tienen los estudiantes del término paz y las formas en que se manifiesta $y$ promueve, diagnosticar las necesidades, expectativas e intereses de los mismos para la implementación de la cátedra de la paz y diseñar y aplicar una propuesta curricular para fomentar la cultura de paz.

2. A los estudiantes participantes en el proceso de investigación, comprender la realidad en que se encuentran, fomentar acciones que promuevan la paz y promover estrategias creativas para el mejoramiento de la convivencia escolar.

Por otro lado, esta investigación también asumió una perspectiva de investigación acción, según Elliott (1993), esta consiste en el estudio de una situación Social para mejorar la calidad de la acción dentro de la misma, y es una reflexión de las acciones humanas que van encaminadas a mejorar y modificar las situaciones que se presenten dentro del aula, pues es netamente aplicada al ámbito educativo.

Atendiendo a las anteriores consideraciones, este diseño permitió analizar la situación social en que tiene lugar esta investigación, mejorar la realidad en que tiene lugar la práctica y hacer un acercamiento al cambio de las acciones que se desean mejorar, en este caso: los procesos de convivencia en la promoción y construcción de una cultura de la paz.

Cabe resaltar que en consecuencia de todas las restricciones que se han presentaron a causa de la pandemia por el COVID-19, y teniendo en cuenta que desde marzo del año 2020 el aprendizaje dejó de ser presencial con la asistencia de los estudiantes las escuelas, para desarrollarlo a través de aulas virtuales o diversas estrategias para garantizar $y$ conservar la salud y la vida de los estudiantes, esta investigación se aplicó de manera virtual mediada por videollamadas a través de la plataforma Microsoft Teams, encuentro sincrónicos programados y otras estrategias en el ámbito de la virtualidad.

A raíz de los cambios vertiginosos en la educación virtual del siglo XXI, se implementa la modalidad del aprendizaje virtual o electrónico conocido en el idioma inglés como e-learning. Sin embargo, Rosenberg (2001) define el aprendizaje virtual como el uso de las tecnologías basadas en internet para proporcionar un amplio despliegue de soluciones a fin de mejorar la adquisición de conocimientos y habilidades. Dicho de otra manera, la educación virtual es una opción más de actualización permanente que responde a las necesidades de cada persona, ofreciendo así diferentes alternativas o soluciones a una serie de situaciones que la escuela convencional no puede atender. No obstante, esta modalidad sigue formando parte de los retos que día a día se asumen en el ámbito educativo y en la sociedad, y sus fundamentos y evolución han permitido que se considere dentro de las ofertas académicas más empleadas en la actualidad.

La institución educativa donde se desarrolló la investigación se encuentra ubicada en el municipio de Tibú (Norte de Santander), una zona que ha sido históricamente afectada por la violencia. Con licencia para desplazar Masacres y reconfiguración territorial en Tibú Catatumbo (2015), y Catatumbo: memorias de vida y dignidad (2018) del Centro Nacional de Memoria histórica son informes que representan la importancia de favorecer los espacios de reconstrucción de los hechos que marcaron el pasado y les garantice a las victimas la reconciliación y reconstrucción de sus daños para conocer la verdad y evitar la repetición de hechos violentos en el futuro, también dichos informes pretenden dar a los entes tanto públicos como privados las competencias necesarias para la adecuada 
atención integral y garantía de los Derechos Humanos de las víctimas.

Por las razones anteriormente nombradas, hablar de memoria histórica implica dignificar la vida de los seres humanos y favorecer la construcción de una paz sostenible en cada uno de los territorios, lo que evidencia la pertinencia de construir desde los entornos escolares lugares de paz que luego van a ser proyectados a la sociedad y al desarrollo de un país más justo e integral.

$\mathrm{La}$ población con quienes se desarrolló la investigación se caracteriza por estar comprendida entre los 13 y 14 años de edad, es un grupo de 34 estudiantes compuesto por 12 hombres y 22 mujeres, quienes desde que cursaban grado segundo primaria en el año 2015 habían presentado una serie de situaciones que no les ha permitido convivir sanamente, pues su trato muchas veces fue con palabras soeces, había poca comunicación y diálogo y hacían uso de la fuerza y hasta de los golpes para resolver un conflicto, para manifestar su inconformidad frente alguna situación, o tomar represalias contra otro compañero por alguna situación. Posteriormente se escogió una población base de 5 estudiantes, con los cuales se desarrolló la esta propuesta y los estudiantes que fueron elegidos se caracterizaron por ser agresores, víctimas de agresiones físicas de sus compañeros o haber sido víctimas de acoso, de intimidación y de rechazo de sus compañeros.

Por lo anterior, aplicar la teoría de resolución de conflictos permitió subsanar las heridas que la violencia escolar había dejado en ellos, permitió mejorar las relaciones de convivencia y proyectar la trascendencia hacia los demás miembros de su grupo para favorecer la armonía y la convivencia escolar.

Con el ánimo de alcanzar los objetivos propuestos y tomando como referencia lo expuesto por Andrade \& Gelvez (2019) los fundamentos teóricos para una buena convivencia en estudiantes de edad escolar está en primera instancia el implementar estrategias metodológicas que promuevan el desarrollo de aprendizajes, en ese sentido, exponen también, que las mismas deben estar acorde a los estilos de aprendizajes presente en cada estudiante atendiendo a la diversidad de inteligencias desarrolladas y sus características socioeducativas para construir conocimientos, asimismo, propician el sentido de la creatividad ya que contribuyen a organizar esquemáticamente ideas para la formación de conceptos contextualizados con la realidad estudiada.

Así las cosas, también resulta valioso aplicar los referentes que enuncian, Marín, Triana, Martínez \& Alzate (2016) al mencionar que la escuela debe hacer intervenciones con familias, para reconocer las pautas de acción que favorecen el proceso de paz y a partir del trabajo comunitario se mitiguen las determinadas formas de violencia y se promuevan procesos de perdón para fomentar la sana convivencia y reconciliación en el marco del postconflicto.

Un claro ejemplo de estrategia para la promoción de la paz y como respuesta a las necesidades de miles de niños afectados por la violencia en Colombia, en el año 2013 el poeta Javier Naranjo diseña el proyecto "Los Niños Piensan la Paz", y en el año 2015, año de su publicación, el autor Gaviria explica que este proyecto recoge las definiciones que más de novecientos niños de todo el país hicieron de la palabra paz y que fue ilustrado por la historietista Powerpaola, quien hace parte del proyecto cultural La paz se toma la palabra, que desde 2013 desarrolla la Subgerencia Cultural del Banco en 28 ciudades colombianas y que busca palabras e imágenes que permitan nombrar e imaginar la paz. Los niños piensan la paz es un proyecto en el que, a través de juegos de palabras y ejercicios de producción de textos, se exploraron los sueños, imágenes y deseos que tienen los niños colombianos sobre la paz.

Para el caso de esta investigación, las estrategias pedagógicas usadas fueron diseñadas de manera que respondieran a los intereses de los estudiantes y fueran lúdicas, innovadoras y de su agrado, por consiguiente, se hizo uso de los siguientes instrumentos para su aplicación:

- Prueba de diagnóstico y de salida. Diseñadas mediante un formulario 
Google tuvieron por intención, la primera: reconocer qué conocimientos o saberes previos tenían los estudiantes acerca del concepto de paz, de conflicto y evidenciar cómo los solucionan cuando se presentan en el aula. Teniendo en cuenta que la unidad didáctica se desarrolló acorde a los lineamientos y competencias propuestas en la cátedra de la Paz para el grado octavo, los objetivos se centraron en el desarrollo y alcance de las competencias y estándares propuestos, comprobando así el nivel de desarrollo logrado por los estudiantes, y su capacidad para tomar decisiones en relación con la planificación y enseñanza de la teoría de resolución de conflictos. Y la segunda, tuvo por función: reconocer junto con la evaluación diagnóstica la apropiación que los conceptos trabajados acerca de paz y resolución de conflictos hubiesen sido apropiados. Esta prueba tiene una pequeña variación con relación a la de diagnóstico, pues se realizaron las mismas preguntas con la intención de corroborar la apropiación del contenido, y sirvieron como recurso pedagógico para ayudar a constituir acciones y planes de mejora para reforzar en el estudiante aquellos conceptos que no fueron correctamente apropiados y fortalecer aquellos que fueron correctamente apropiadas.

- Unidad didáctica. diseñada para el desarrollo de la teoría de resolución de conflictos de Galtung (1985) con los estudiantes de grado octavo acorde a los lineamientos que propone el Ministerio Educación Nacional en la guía \#6 FORMAR PARA LA CIUDADANÍA SÍ ES POSIBLE, tomando como referentes los desempeños del núcleo convivencia y paz que proponen para el grado en mención.

- Página WEB FANZINEROS POR LA PAZ. Es una página web creada bajo el dominio de WIX, autoría de Dayanna Zuley Cely Fuentes, investigadora del presente proyecto. Esta página web se encuentra organizadas en 8 sesiones de trabajo y dentro de cada sesión se encuentran los contenidos que se van a desarrollar en cada uno de los encuentros. Los contenidos están estrechamente relacionados a paz, resolución de conflictos, comunicación, trascendencia del ser humano y otros temas que son relevantes e importantes en el desarrollo de la propuesta de investigación. La razón principal de ser de esta página es hacer asequible a los estudiantes la información y el material que se va a utilizar en cada uno de los encuentros pedagógicos aprovechando los entornos virtuales ofrecidos en la institución.

- Cartilla MI DIARIO. Es una adaptación de la cartilla original propuesta por UNITAR y la Ciudad Don Bosco, un programa desarrollado en la ciudad de Medellín Colombia para liderar procesos de construcción de paz y ciudadanía en poblaciones con condición de vulnerabilidad. En esta cartilla se propusieron actividades que los estudiantes realizaron luego de haber analizado, estudiado $y$ apropiado los temas orientados desde la página web. Está diseñada en un formato digital e impreso tamaño carta $(21 \mathrm{~cm} \mathrm{x} 27 \mathrm{~cm})$ en el aplicativo CANVA, pues este permite un formato agradable a la vista, favoreciendo las necesidades $y$ expectativas propias de la edad de los estudiantes con los que se desarrolló la propuesta.

La investigación se desarrolló teniendo en cuenta 8 fases propuestas mediante las actividades descritas en la Tabla 1: 


\section{Tabla 1}

\section{Sesiones de trabajo para el desarrollo de la propuesta didáctica}

\section{Sesión Actividad}

1

1. Presentación del tema: la violencia y sus tipos, apoyo en dispositivas y videos a través de encuentros virtuales de la plataforma institucional TEAMS.

2. Actividad: la maleta, dibujo en ella los elementos que considero necesarios llevar para vivir en un mundo sin violencia.

Actividad del iceberg cómo me veo, y cómo me ven los demás.

3. Realizo mi autorretrato cómo me veo y cómo me ven los demás.

1. Presentación del tema: disputa, dilema y conflicto.

2. Presentación del tema cómo transformar conflictos según Galtung (2003), La cooperación armoniosa para transformar el conflicto:

3. Actividad el camino: dibujar en un camino pedregoso las situaciones u obstáculos que no nos permiten tener una sana convivencia en el grupo y que genera conflicto entre ellos.

4. Actividad el bombillo: decorar un bombillo, y escribir alrededor de él, las situaciones o acciones que le permitirán al grupo mejorar sus relaciones y erradicar los conflictos.

5. Actividad la montaña: dibujarse a sí mismos y a su grupo al iniciar la montaña, y en la cima la palabra paz, en la montaña deberán escribir las acciones que nos permitirán lograr la cima.

1. Charla: la capacidad de escucha y la mediación con Sol María Macías, psicóloga, apoyo en dispositivas y dinámicas mediante encuentro virtual de la plataforma institucional TEAMS.

2. Características del facilitador de conflictos y Elección del facilitador de conflictos.

3. Mesa redonda: llegar juntos a soluciones trascendentes y de compromiso frente a los conflictos tratados la semana anterior.

4. Actividad lluvia de ideas: registrar las opiniones de mis compañeros.

1. Desarrollo del tema: creatividad, video que es la empatía y cómo desarrollarla, apoyo en dispositivas y videos a través de encuentros virtuales de la plataforma institucional TEAMS.

2. Significatividad de la creatividad y la empatía: la valoración de la vida y del otro en la resolución de conflictos.

3. Actividad: fomentar la calma a través del coloreado de mándalas

4. Actividad: el mapa de la empatía, dibujarse a sí mismos y registrar los valores o actividades que me permiten fortalecer la empatía.

1. Desarrollo del tema y conceptos de perdón, reconstrucción y reconciliación según Galtung (2016), apoyo en dispositivas y videos a través de encuentros virtuales de la plataforma institucional TEAMS.

52 2. Actividad la caja: dibujar en ella o escribir lo que reconozco lo que me hiere.

3. Actividad el termómetro de las emociones: escribir o dibujar en el termómetro las cosas que te llevan a los estados emocionales: furioso, enojado, frustrado, calmado y feliz.

4. Actividad el perdón: reconocer a alguien que me ha herido o ha hecho daño y escribirle un mensaje, responder ¿esta situación cómo cambiaría nuestras vidas?

1. Diálogo: el ser y la trascendencia.

2. Actividad el mensaje de la gratitud: escribir un mensaje a alguien especial

$6 \quad 3$. Actividad: reconocer lo importante en mi vida y a lo que debo darle espacio

4. Actividad: reconociendo mis metas

5. Actividad la escalera: 10 escalones con cosas que puedo hacer para lograr mi meta

6. Actividad: cómo me veo en el futuro

1. Conceptualización del tema: la paz

2. Presentación de 5 personajes que han ganado premio nobel de paz

3. Cómo se educa en Colombia para la paz, apoyo en dispositivas y videos a través de encuentros virtuales de la plataforma institucional TEAMS

4. Reflexión de Mahatma Ghandi: no hay camino para la paz, la paz es el camino.

5. Actividad: construir el decálogo de nuestros derechos, como hacerlos valer y respetarlos.

6. Actividad: promoviendo la protección al medio ambiente

1. Charla de cierre, apoyada en encuentro virtual a través de la plataforma institucional TEAMS. Responder:

- ¿Qué aprendí?

- ¿Qué actividades me gustaron?

- ¿Cómo cambió mi vida?

Nota. En la tabla se visualizan las estrategias pedagógicas usadas fueron diseñadas, elaboración propia (2021). 


\section{Resultados}

En el aula de clase anteriormente se evidenciaban conductas agresivas, el uso de palabras soeces, disputas y conflictos, los cuales se fueron disipando con el reconocimiento de las formas de violencia y mejorando los procesos de comunicación y diálogo cuándo se presentase un conflicto. Así mismo, los estudiantes, reconocieron la importancia de hacer uso de la comunicación para resolver un conflicto, socializar las posibles soluciones a estos, superar todas las barreras que impedían el desarrollo de una sana convivencia y promover el desarrollo de una cultura de paz, para llevar a cabo lo anterior, se procuró dicho en palabras de Lederach (2000) trabajar sobre los problemas concretos que tienen las partes en conflicto, centrarse en las preocupaciones y necesidades de cada uno y establecer un ambiente de negociación y perdón.

Implementar la figura del facilitador de conflictos, quien fue destacado y elegido por los mismos estudiantes por su cualidades pacíficas y vivencia de valores, favoreció los procesos de comunicación para solventar las situaciones de conflicto que se presentaron dentro del aula, garantizando en todo momento espacios para la escucha, mediación y resolución de conflictos, pues según Carballeira (2018) el diálogo es la mejor herramienta para resolver los problemas que implican a personas y sentimientos, y al hablar de dialogo en una situación de conflicto es importante destacar la figura del mediador, para ayudar a ser más llevaderas las disputas entre las personas que conforman el conflicto.

En este orden de ideas, el facilitador de conflictos era quien daba entrada al diálogo y establecía formas de reconstrucción de conflictos, manteniendo las mediaciones entre los implicados a través de la vivencia de la democracia, en donde se analizaban los argumentos del agresor con la víctima para no incrementar los niveles de agresividad, y en donde la víctima pudo sentirse complacida por la acción correctiva que se aplicó a su agresor.
Por otra parte, con los estudiantes que se aplicó la investigación, se favoreció la creación de un espacio que promoviera los procesos de reconstrucción y documentación de la memoria, de resiliencia, de conciliación de conflictos y de crear una historia nueva, también, se convirtió en un factor de desarrollo y convivencia al abrir espacios de diálogo y discusión en torno a la construcción conjunta de la paz, contribuyendo así al restablecimiento de la cultura de la paz, entendida como la apropiación de conocimientos y competencias ciudadanas para la convivencia pacífica, la participación democrática, la equidad, la pluralidad y el respeto por los Derechos Humanos

En resumen, y contrastando los resultados de esta investigación con los aportes de ÁlvarezOvallos, Gélvez-López \& Mosquera-Téllez (2020) es preciso confirmar que la convivencia escolar es un aspecto fundamental del proceso formativo, en tanto no solo enseña a convivir, sino permite descubrir habilidades, demostrar valores y generar espacios interactivos de respeto, armonía y paz, esenciales para lograr los propósitos de aprendizaje.

\section{Conclusiones}

Para facilitar una cultura de paz y educación por medios pacíficos en la escuela, fue muy apropiado garantizar que cuando se presentara un conflicto inmediatamente se activara la ruta de trabajo en donde hiciera intervención el trabajador por la paz, se facilitaran los procesos de diálogo, de escucha de la víctima y del agresor, se garantizara la democracia y la equidad, se diera primacía a la reconciliación, se repararan los daños en caso de ser necesario, se firmara un contrato de aceptación y reconciliación y hubiese perdón de parte de la víctima hacia el agresor para favorecer la no repetición del conflicto.

Tratando de profundizar en el desarrollo de una cultura de paz en la escuela, es de vital importancia dentro del ámbito educativo pensar, comprender $\mathrm{y}$ aprender de las realidades de las escuelas con relación a los contextos de guerra en donde han sido afectadas por la presencia de las víctimas y 
victimarios, también por las condiciones de desigualdad económica y social que se han venido profundizando con la implementación de las políticas neoliberales durante los últimos veinte años, sobre todo para imaginar y edificar la escuela en el posconflicto, con presencia creativa en la formación del pensamiento crítico y del ciudadano comprometido en la lucha política, social, cultural y pedagógica, teniendo en cuenta el reconocimiento de las víctimas, por la construcción de la paz con democracia integral, justicia social, dignidad humana y el Estado social de derecho.

Además, con esta propuesta no solo buscó armonizar a las relaciones entre los estudiantes de la institución para que los conflictos sean transformados de forma creativa y pacífica, sino que se asumió el reto de ahora en adelante de consolidar una cultura de paz mediante la comunicación asertiva, favoreciendo las relaciones de convivencia y equidad, es decir que, no sólo se brindaran las orientaciones necesarias para resolver situaciones conflictivas, sino que se diera importancia para generar en la comunidad educativa la capacidad de diálogo, el respeto, la tolerancia, la solidaridad y otros aquellos valores que aporten a la conformación de una cultura de paz, en donde no haya lugar para la violencia pero si para el trabajo cooperativo, el dialogo y para garantizar la trascendencia del ser humano.

Finalmente, y retomando los postulados de Zurbano, (1998) la educación para la paz debe preparar al individuo para que procure la armonía en las relaciones humanas en todos los niveles, incluyendo la concientización y la búsqueda de soluciones concretas a sus conflictos. Para ello, los actores deben reconocer la importancia de educar desde las primeras edades en las normas de convivencia y de este modo construir conocimientos (en casa, la escuela y los lugares públicos) basados en las experiencias personales y sociales que preparen a las nuevas generaciones para vivir en paz, en una sociedad con mayores cotas de justicia.

En conclusión, la educación para la paz podrá revelar que los conflictos son oportunidades educativas para aprender a construir otro tipo de relaciones y preparar a los estudiantes para la vida, aprendiendo a hacer valer y respetar sus derechos de una manera no violenta.

\section{Referencias}

Álvarez-Ovallos, A., Gélvez-López, A. \& Mosquera-Téllez, J. (2020). Conflicto Escolar en la Educación Rural del Nororiente de Colombia. Revista Tecnológica Educativa Docentes 2.0, 9 (2), 5-15. https://doi.org/10.37843/rted.v9i2.135.

Andrade, A., \& Gelvez, A. (2019). Propuesta metodológica para potenciar procesos de convivencia, en los estudiantes de quinto grado de la sede $\mathrm{N}^{\circ} 2$ en la institución educativa Manuel $\mathrm{j}$ del Castillo de Ciénaga magdalena. Revista Tecnológica-Educativa Docentes 2.0,7(2), 60-65. https://ojs.docentes20.com/index.php/revistadocentes20/article/view/12

Carballeira, E. (2018). El diálogo, la mejor herramienta para resolver conflictos. Servicios sociales 16, (1), https://www.aucal.edu/blog/servicios-socialescomunidad/resolver-conflictos-mediante-el-dialogo/

Centro Nacional de Memoria Histórica. (2015). Catatumbo: Memorias de vida $y$ dignidad. Editorial Panamericana.

Centro Nacional de Memoria Histórica (2018). Con licencia para desplazar. Masacres y reconfiguración territorial en Tibú, Catatumbo. Editorial Panamericana.

Chaux, E., Lleras, J. \& Velásquez, A.M. (2004). Competencias ciudadanas: de los estándares al aula. Una propuesta de integración a las áreas académicas. Editorial Universidad de los Andes.

Elliott, J. (1993). El cambio educativo desde la investigaciónacción. Editorial Alertes.

Galtung, J. (1984) ¿Hay alternativas?: cuatro caminos hacia la paz y la seguridad. Editorial Fontamara.

Galtung, J. (1985). Sobre la paz. Editorial Fontamara.

Galtung, J. (2003). Paz por medios pacificos: paz y conflicto, desarrollo y civilización. Editorial Bakeaz,

Galtung, J. (2016). La violencia: cultural, estructural y directa. Editorial Fontamara.

Gaviria, P. \& Naranjo-Moreno, J. (2015). Los niños piensan la Banco de la República de Bogotá.

paz.

Marín-Hinestroza I., Triana-Osorio, L. A., MartínezSaldarriaga, M. G. \& Alzate-Berrio, S. M. (2016). Perdón, convivencia y reconciliación en el proceso de paz, desde una mirada psicológica. Revista Poiésis (31). 245-256. 
Lederach, P. (2000). El Abecé de la paz y los conflictos: educación para la paz. Editorial Catarata.

Rosenberg, M. J. (2001). E-learning: strategies for delivering knowledge in the digital age. Editorial McGraw-Hill.

Zurbano, J. (1998). Bases de una Educación para la Paz y la Convivencia. Editorial Fondo de Publicaciones del Gobierno de Navarra. 